\title{
BIO.I0 - Genetic algorithm for deimmunization of Escherichia coli L-asparaginase
}

Raphael Trevizani ${ }^{1 \star}$.

1 Fiocruz - Fundação Oswaldo Cruz.

Introduction: L-asparaginase is a heterologous enzyme expressed in Escherichia coli and used in leukemya therapy that triggers an immune response due to the presence of linear T-cell epitopes within the protein sequence. This results in the sustained production of antibodies that causes hypersensitivity problems and in the neutralization of the therapeutic effect. To diminish the potential immunogenicity, the protein can be subject to a process of epitope deletion via genetic manipulation of key MHC-high-binding residues. As an exhaustive evaluation of the mutants is prohibitive for experimental scientist because the number of variants is too big, in silico tools can be used to diminish the search space.

Objective: This work aimed to use in silico tools to propose deimmunized variants of E.coli $\mathrm{L}$-asparaginase via manipulation of key aminoacids in the protein sequence.

Methodology: Linear epitopes were mapped and scored with the TEPITOPE predictor. The score given by TEPITOPE is used as an indication of the likelihood of protein immunogenicity. A genetic algorithm was used to evolve and evaluate the best variants of a deimmunized L-asparaginase. The initial population of the genetic algorithm consists of a set of 30 identical proteins, and in each generation, random residues of the epitopes are substituted by a set of selected aminoacids. In each generation, two proteins are randomly chosen and a crossover operation is performed, creating two new mutants. The mutants with the highest immune score are eliminated from the population, which allows the mutations that diminish the immune score to accumulate in the best proteins. As every mutation inserted has the potential to compromise the structural stability of the protein, for each mutant generated the folding $\mathrm{dG}$ is estimated using the software FoldX. The folding dG must not differ in $0.5 \mathrm{kcal} / \mathrm{mol}$ when compared to the native, otherwise, the mutant is eliminated because it is considered unstable. The preferred mutants have the lowest immunogenicity possible with the fewest number of mutations. A Pareto approach is used to narrow the set of variants to those that represent the best trade-off between immunogenicity and number of mutations.

Results: In this work, a genetic algorithm was used to generate deimmunized variants of E.coli L-asparaginase. Among the 51216 mutants evaluated, 14 represented the best tradeoff between immunogenicity and low number of mutations. The genetic algorithm proposed was successfully used to redesign the immunogenic regions of the therapeutic L-asparaginase and generated variants predicted to outperform previous experimental efforts. This confirms the capacity of the algorithm to generate tens of thousands of unique mutants of therapeutic interest.

Conclusion: Protein engineering is an invaluable tool to improve the therapeutic importance and circumvent the immunogenicity of commercial L-asparaginase for an improved, patientstailored therapy.

Keywords: L-asparaginase; Deimmunization; Therapeutic proteins 